\title{
III. On the electric discharge in gases
}

\section{Eilhard Wiedemann}

To cite this article: Eilhard Wiedemann (1884) III. On the electric discharge in gases, Philosophical Magazine Series 5, 18:110, 35-54, DOI: 10.1080/14786448408627562

To link to this article: http://dx.doi.org/10.1080/14786448408627562

$$
\text { 册 Published online: } 29 \text { Apr } 2009 .
$$

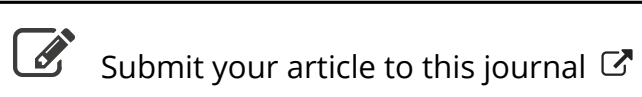

\footnotetext{
Џll Article views: 3
}

Q View related articles $\sqsubset$ 
So nearly complete is the separation at $25^{\circ}$, that above this temperature the apper stratum gives more in volume by expansion by heat than it loses by the rise of the plane of demarcation. To get an idea of the completeness of the separation at $50^{\circ} \mathrm{O}$., we may compare the volume of the lower stratum at $50^{\circ}$, which is 8.0 , with that of 7.885 grams of water at $50^{\circ}$, which is $7 \cdot 93$. This signifies that at $50^{\circ}$ the lower stratum must be nearly pure water, and the upper nearly pure ethylamine. For the plane of separation stands only $\frac{1}{80}$ of the height of the original water-column above that height. See fig. 1 , curve $b$.

[To be continued.]

\section{On the Electric Discharge in Gases. By Eulmard Wiedemann.*}

[Plate II.]

$\mathrm{N}$ a number of previous communications $\dagger \mathrm{I}$ have investigated the behaviour of gases under the influence of electric discharges in various ways. The present research is a continuation of those investigations, and is concerned with (1) the effect upon the phenomena of interposing resistances between the poles of the machine and the electrodes of the discharge-tube ; (2) the phenomena obtained with different distances between the electrodes; (3) measurements of the heating effect upon the gas at different points of the discharge ; (4) deflection of the positive discharge ; (5) the behaviour of the discharge under the influence of the magnet; $(6)$ the examination of an hypothesis as to the nature of the kathode-rays, as well as (7) the nature of the positive discharge and the stratifications; (8) further observations on the influence of the magnet; (9) behaviour of bad conductors as kathodes; (10) connexion of the potential of the discharge with the form of the electrodes ; and (11) remarks on the management of electrophoric machines.

The great complexity of the problem explains why the separate phenomena have not been hitherto sharply distinguished from each other, as, for example, the "glow" appearing at somewhat higher pressures, the kathode-rays which are formed at lower pressures, \&c.

* Translated from the Annalen der. Physik und Chemie, vol. xx. p. 756 (1883), with additions and conrections by the Author.

$\dagger$ Wied. Ann. v. p. 200 (1878); vi. p. 298 (1879); ix. p. 157 (1880); x. p. 2022 (1880); 1 lill. Mag. vol. x. p. 35̃7. The glass apparatus has beell made for the experiments by Herr Götze.

D 2 
There are also a series of interesting facts which have been clearly observed, but of many of which it was not possible for the same reason to determine the intimate connexion, by which remark it is of course not intended in any way to depreciate the work of the earlier investigators.

The separate details have only gradually become of importance, and even now it is often difficult to recognize them accurately in descriptions. I have endeavoured in the subjects of which I treat to distinguish these relationships as accurately as possible, and to make clear the conditions under which the phenomena oceur.

In order to fix ideas we will agree upon the following nomenclature:-The phenomena are best observed in tubes having electrodes of a flat shape, because then the different stratifications are formed at right angles to the axis of the tube.

If $k$ (fig. 3 ) is the kathode, scarcely any light is to be seen in the part of the tube next it-this we will call the dark negative space; next to this comes a stratification (b), sharply bounded on the side next the kathode, the bright negative layer. From it issues light $(b p)$ towards the anode, which becomes continually fainter; this we will call the glow-rays. They are separated from the positive column of stratified light, of which the first layer is $p$, by a dark space $h p$ which may be called the dividing space. The whole is traversed by the kathode-rays $l m$ issuing from the kathode $k$.

1. Influence of Interposed Resistances.-The experiments were so arranged that the one pole of the electrophoric machine was connected with the earth, whilst the other was in connexion with one electrode of the tube, the other electrode being connected through the coils of a galvanometer with the earth. Resistances could be introduced either between the dischargetube and the machine, or between the discharge-tube and the earth. They consisted of long glass tubes on the outside coated with shellac, and filled with distilled water in which conducting wires plunged. The discharge was observed in a mirror rotating with moderate velocity.

The result generally obtained was that, whether resistances were included or not, the deflection of the galvanometer was the same during uniform action of the machine, and that consequently the quantity of electricity passing through the discharge-tube in unit time was always the same.

The number of discharges, however, was found to be considerably greater when resistances were employed than without, and the same result was obtained at very different pressures. With discharge-tubes of the form usual to spectral-tubes the discharge is always discontinuous. With wider discharge-tubes 
and flat electrodes, and when the gas has the density for which only a small potential is necessary for the discharge, the image in the mirror often appeared continuous when resistances were included, and, when they were removed, resolved itself into a series of separate images.

With discontinuous discharges, whether with or without included resistances, in both cases the result obtained * by G. Wiedemann and R. Rühlmann held good:-

"If the positive electrode is connected with the machine, and the negative put to earth, the number of discharges is always smaller than with the reverse mode of connexion; the quantity of electricity necessary to bring about a discharge is therefore greater in the former case than in the latter."

It is a matter of indifference which of the two poles of the machine is positive and which negative. A reversal of the poles of the machine can always be produced by simply stopping the machine and starting it again.

The influence of the introduction of resistances may be explained as follows :-

To bring about a discharge from a metallic electrode, the electricity must be accumulated upon it with a perfectly definite density. If quantities of electricity are present upon conductors connected with the electrode, these may, when once the discharge has begun, and if they can reach the electrode quickly enough, leave the electrode at a smaller potential than is actually necessary for the commencement of the discharge. Such accumulated electricity is, however, present in the larger electrophoric machines in a high degree, and in the smaller machines in a smaller degree. To the conducting wires to the poles and to the combs of the machine and the neighbouring parts of the glass plates there are, between any two discharges, considerable quantities of electricity given off, which, as soon as discharge from an electrode has been established, flow to it and continue the discharge through the discharge-tube. If the connexions are metallic, the flow is much more rapid ; the time required to pass from the machine to the electrode is small in comparison with the definite, although immeasurably small, interval of time occupied by the discharge, and the whole quantity of electricity passes through at once. But at the same time the density upon the whole conducting system in comnexion with the electrode sinks at once to zero; and a longer time must elapse until it is again raised by the machine so high that a new discharge can take place. If we include larger resistances, the electricity will only flow slowly to the electrode when once the discharge has taken place. The * Pogg. Ann. cxlv. pp. 235, 344 (1872). 
density sinks therefore very rapidly at the electrode, and only small quantities of electricity can follow the first quantity. But the electricity accumulated upon the machine and flowing to the electrode will very soon raise the density to the necessary point for the discharge to take place again, and without large quantities of fresh electricity being produced. The number of the discharges must therefore in the latter case be mnch greater than in the first, with the same average intensity of current, since in the latter case, in each discharge only the quantity of electricity upon the electrode and close to it is discharged, and in the former, on the contrary, the quantity existing upon a much larger surface.

The machine itself therefore acts as a sort of feeble condenser.

But since the law relating to the difference between positive and negative electricitiy, discovered by my father, holds good both when resistances are included and without, his result cannot be explained by irregularities in the action of the machine, by different behaviour of the combs, and so on, since these would be compensated by the condensing action of the machine. We may call the discharge, when resistances are included, the normal discharge ; and the quantity of electricity which passes over, under the same circumstances, also the normal quantity, because it is just that quantity which, under the precise conditions of the experiment, must be accumulated on the electrode in order to bring about a discharge.

Since we observe, further, that always when, without resistances, the discharge is unstratified, it becomes stratified if resistances are included, we may also conclude that "the stratified discharge corresponds to the normal discharge;" and since the stratifications evidently require for their formation a regular discharge, it further follows that in the normal discharge the discharges occur in their simplest form.

In exact agreement with the above we find that, when once stratifications have been formed, whether with or without resistances, these disappear as soon as an air-spark is included, the quantity of electricity transmitted in each discharge being thus increased. Numerous experiments showed that, as a rule, the stratifications form best when the negative electrode is connected with the machine, and the positive with the earth through a large resistance. In the case of deviations of this rule, which certainly occur frequently, it was always found that the discharges, which were most numerous under the conditions noted, gave place to less numerous discharges. The reason of this could not, amongst numerous disturbing causes, always be certainly determined : thus, for example, very peculiar pheno- 
mena occur when the machine is made to work vory slowly, and consequently the supply of electricity is much reduced. If the pressure is so low that the dark negative space is about 2 centim. in breadth, whilst the glow-ravs are separated from the positive discharge by the dividing space, or even if there is no positive discharge to be seen in the tube, and if then the machine is worked more and more slowly, the positive light expands round the positive electrode, whilst the negative light also expands considerably, until the positive and negative luminosities meet. This condition may be maintained for any length of time.

Since the experiments described above had shown that the discharges obtained simply from an electrophoric machine represent the simplest mode of discharge, it was necessary to investigate whether the propositions first stated by G. Wiedemann and R. Rühlmann* and myself $f$ as to the independence of the heating of the gases upon the width of the tube, and the quantity of electricity traversing it, would also hold good for the case in which, by removing resistances, the quantity of electricity passing in each discharge is increased. If it is shown that changing the resistances produces no considerable effect upon the quantity of heat produced either in a wide or in a narrow tube, then it follows that if these two tubes are included in the same circuit, equal quantities of heat must be produced per unit of length, independent of the cross-section of the tube.

The experiments are arranged exactly like those described in a previous communication $\neq$. In these experiments the whole quantity of heat produced and that evolved within the space connecting the electrodes (in the case of spectral tubes within the capillary part) were determined. Experiments on the heating of the electrodes themselves will follow later.

Let $p$ denote the pressure, $m \mathrm{~W}$ denote with included resistances, $o \mathrm{~W}$ without resistances, $\mathrm{F}$ that no resis nce but a spark 5 millim. long was included; + or - that th positive or negative electrode of the machine, as the case may be, was connected with the tube, 0 that both were connected with the machine; $x$ as before denotes a very low pressure, and $x x$ a still lower pressure. The numbers under,,+- 0 are numbers which are proportional to the quantities of heat evolved in the unit time by equal currents. They represent here only the relative, not the absolute values.

* Pogg. Ann. cxlviii. pp. 35, 252 (1876).

+ Wied. Ann. x. p. 202 (1880); Phil. Mag. vol. x. p. 357.

$\ddagger$ Ibrid. 
Whole quantity of heat evolved.-The discharge-tube consisted of two small bulbs of 12 millim. diameter, connected by a capillary tube of 40 millim. length.

\begin{tabular}{|c|c|c|c|c|c|c|c|c|c|}
\hline \multicolumn{4}{|c|}{$p=9.75$} & \multicolumn{3}{|c|}{$p=1.9$} & \multicolumn{3}{|c|}{$p=x$} \\
\hline & + & - & 0 & + & - & 0 & + & - & 0 \\
\hline$m \mathrm{~W}$ & $\ldots$ & $\ldots$ & $2 \cdot 92$ & $3 \cdot 11$ & 3.08 & $\ldots$ & 7.33 & 6.59 & \\
\hline$\circ \mathrm{W}$ & $\ldots$ & $\ldots$ & 327 & 352 & 3.23 & $3 \cdot 43$ & 8.36 & 6.86 & 6.54 \\
\hline F & $\ldots$ & $\ldots$ & $10 \cdot 10$ & $5 \cdot 0$ & 5.08 & $\ldots$ & $12 \cdot 31$ & & \\
\hline
\end{tabular}

From these numbers we see that the total heating does not alter much, although a small increase is perceptible when the $\operatorname{sam} \theta$ electricity is transmitted in a decreasing number of discharges. The removal of resistances corresponds to the including of an air-spark, but the heating is certainly not greater in proportion to the smaller number of discharges. The same considerations which I have already stated * therefore hold good.

Heat evolved in the capillary connecting tube.

(1) Heating in a capillary of 1 millim. diameter :-

\begin{tabular}{|c|c|c|c|c|c|c|c|c|}
\hline \multicolumn{3}{|c|}{$p=12.9$} & \multicolumn{2}{|c|}{$p=2}$. & \multicolumn{2}{|c|}{$p=x$} & \multicolumn{2}{|c|}{$p=x x}$. \\
\hline & + & - & + & - & + & - & + & - \\
\hline$m \mathrm{~W}$ & $10 \cdot 8$ & $13 \cdot 0$ & 4.5 & $5 \cdot 35$ & 2.52 & 1.90 & $2 \cdot 0$ & $1 \cdot 46$ \\
\hline $0 \mathrm{~W}$ & $11 \cdot 5$ & 9.7 & $5 \cdot 13$ & $6 \cdot 10$ & 1.73 & $2 \cdot 64$ & 1.8 & 1.95 \\
\hline F $\ldots .$. & .. & $\ldots$ & $\ldots$ & $\ldots$ & 3.85 & $4 \cdot 20$ & 3.36 & $4 \cdot 0$ \\
\hline
\end{tabular}

Here also the heating with and without resistances is nearly the same, and again the heating increases somewhat as the number of discharges decreases. This is pretty regularly the case when we consider only the negative discharges; with the positive on the other hand, the minimum heating is at low pressures without a resistance. The positive discharge exhibits therefore here, as in many other cases, a less regular behaviour.

(2) Heating in a tube of 4 millim. diameter :-

\begin{tabular}{|c|c|c|c|c|c|c|}
\hline \multicolumn{3}{|c|}{$p=12 \cdot 7}$. & \multicolumn{2}{|c|}{$p=6 \cdot 4,5 \cdot 9$} & \multicolumn{2}{|c|}{$p=6.4,5.9$} \\
\hline$m \mathrm{~W} \ldots$ & + & & $3 \cdot 5$ & $\ldots$ & $6 \cdot 4$ & \\
\hline$o \mathrm{~W}$ & 8.3 & $11 \cdot 1$ & $4: 5$ & $\ldots$ & $6 \cdot 4$ & 63 \\
\hline$F \quad \ldots \ldots$ & $\ldots$ & $\ldots$ & $\ldots$ & 6.8 & $\ldots$ & $9 \cdot 0$ \\
\hline
\end{tabular}

* Wied, Ann, xi, p. 218 (1883). 


\begin{tabular}{|c|c|c|c|c|}
\hline \multicolumn{3}{|c|}{$p=0 \cdot 8$. } & \multicolumn{2}{|c|}{$p=x}$. \\
\hline & + & - & + & - \\
\hline$m W$ & $1 \cdot 6$ & $1 \cdot 6$ & 06 & 0.8 \\
\hline$o W$ & $1 \cdot 3$ & $1 \cdot 6$ & $1 \cdot 0$ & 0.8 \\
\hline$F \ldots$ & $2 \cdot 0$ & $2: 4$ & $1 \cdot 5$ & $1 \cdot 2$ \\
\hline
\end{tabular}

The result is here generally the same as with the narrower tubes. With a decreasing number of discharges the heating for equal quantities of electricity increases relatively very little. But if we take the numbers generally and observe that, especially at low pressures, the including of resistances increases the number of discharges immeasurably, we see that upon including resistances :-

(1) The evolution of heat in a gas by a given quantity of electricity which traverses it is almost independent of the circumstance whether it traverses in one discharge or in many.

(2) Hence, and from the previous result, it follows at once that with two tubes of different diameters, included one behind the other in the same circuit, equal quantities of heat per unit of length are evolved, whether the number of discharges be increased by inserting resistances or not.

In fact the amount of heating in the narrow and in the wide tubes is scarcely changed by the introduction of resistances ; and since they are the same when no resistances are present, they must also be equal in the other cases. Hence the same laws hold for the normal discharge as for that usually examined.

In connexion with this the following remarks must be made. Certain advantages possessed by electrophoric machines over induction-coils and large valvanic batteries for the investigation of the discharge in $\mathrm{v}$ suous tubes, have been noted by various observers, and in particular by my father and by myself. Onr opinion is based upon the fact that, with the electrophoric machines, only the quantity of electricity is discharged each time which is required to produce the potential necessary, under the conditions existing in the tube and at the electrodes, to initiate the discharge.

That these views are not altogether correct is seen from the above ; nevertheless for a number of investigations, and particularly for those of spectroscopic nature, electrophoric machines have advantages over other sources of electricity, not only over induction-coils but also galvanic batteries, since with these it is never possible to transmit large quantities of electricity in disruptive discharge without condensers, by the use of which the conditions are much complicated. With the elec- 
trophoric machine the simple introduction of an air-spark is sufficient.

2. Phenomena at different Distances of the Electrodes.-It is of importance to be able to trace the phenomena which result when the distance of the kathode from the anode is changed within somewhat wide limits. It is essential that the vessel containing the electrodes should not be opened, for with each such opening the pressure would be altered : and the pressure under which the first determinations were made is, especially when low pressures are employed, not to be obtained again.

In order to carry out experiments in the direction indicated, I have constructed the following apparatus (Plate II. fig. 1):-

A is the discharge-tube, which communicates with the airpump at $\epsilon ; \alpha$ is an electrode firmly melted in, which is connected with the machine in the manner already described *. A second electrode, insulated in the usual way, is inserted at $\delta$; the lower part has melted on to it the outer portion $(e)$ of a ground glass joint. The inner portion $(d)$ of the same forms one end of a U-tube, B C, whose longer limb B is about 900 millim. long, and its shorter limb $\mathrm{C}$ about 500 millim.; at the bend it is provided with a tap, and a platinum wire is melted in at E. The two portions of the joint are connected by caoutchouc bands at $s s^{\prime}$ and $t \dot{t}^{\prime}$ in the manner already described $\dagger$. So much mercury is next introduced into the U-tube that it stands in each limb about 400 millim. high.

The second electrode is formed by the plate $\beta$, which can be replaced by a point \&c. It is fastened and connecter with the machine as follows:-A thin platinum wire is melted into one end of a thin glass tube of suitable length, and the end within the glass tube covered with a little mercury. Into this dips the end of an aluminium wire which traverses the whole length of the tube, and on the top of which the plate is screwed. By this arrangement the whole conducting wire up to the electrode was covered with glass. In other cases the metallic wire was left uncovered.

The rod carrying the electrode thus floated with its lower end in the mercury in the limb B. By pouring mercury into $\mathrm{C}$, or allowing it to run out from $h$, the level could be raised or lowered, and thus the electrode $\beta$ made to approach or recede from the plate $\alpha$. To regulate the motion it was necessary to provide the rod with some guidance: the simplest plan was apparently to make the tube $B$ so narrow that the rod can just move up and down it ; there was, however, so powerful a capillary action, that not only in this case, but also when much

* Wied. Ann. x. p. 206 (1880); Phil. Mag. vol. x. p. 360.

$\dagger$ Ibid. p. 209 (1880); Phil. Mag. vol. x. p. 362. 


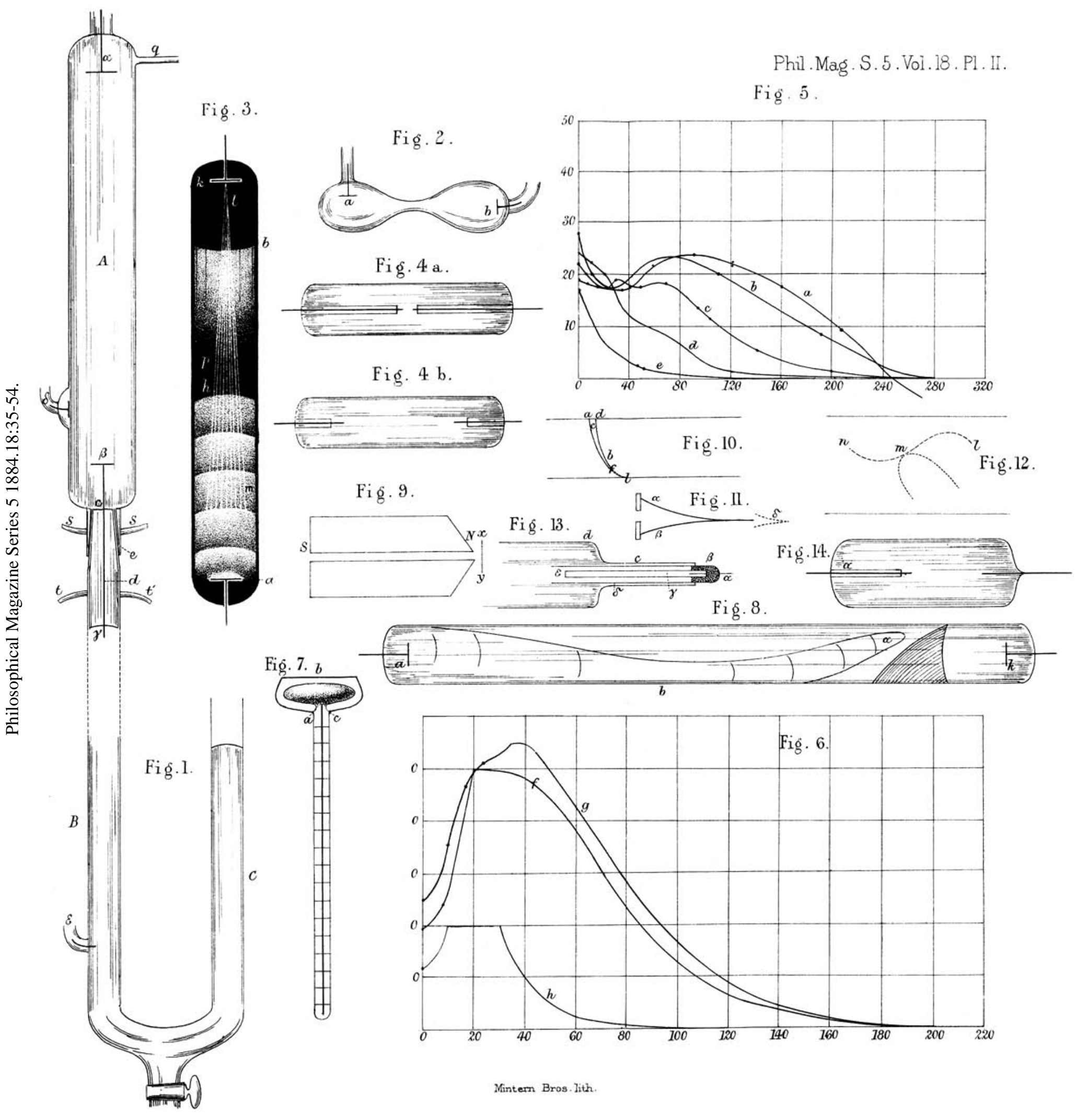


wider tubes were chosen and the rod came into contact with the sides of the tube, any regular notion was impossible. A star-shaped piece of platinum foil was therefore fastened to the lower part of the rod with a little wax; and as a further guide the upper end of $d$ was covered with a plate having in it a hole just large enough to allow the rod to pass through. In order to introduce the electrode $\beta$ into the space $A$, either the tube $d$ was made larger than the plate, or else the tube A was cut in two ; and after the plate had been introduced into $A$ and the rod carrying it passed through $\mathrm{C}_{3}$ the tube was melted together again; the terminal wires of the machine plunged in $\alpha$ and $\epsilon$, or in $\alpha$ and $\delta$, as the case might be.

In order to follow the rhythm of the discharges in the discharge-tube, which it is difficult to do in the luminous phenomena of the tube itself, on account of its great width, and especially when the discharges are irregular, or are confined to special parts of the tube, it is advisable, besides the wide tube, to include also a second tabe in the circuit, which it is best to take of the form shown in fig. 2. This is made out of a wide tube by contracting the middle portion before the blowpipe; the tube is exhausted so far that it offers as small a resistance as possible to the current traversing it: $a$ and $b$ are flat electrodes which offer a smaller resistance to the currents than points do ; they are placed as shown in the figure, in order to afford a rapid indication of the direction of the current. The midale portion of the tube is observed in the revolving mirror; its relatively great luminosity and small section render it easy to judge in the rotating mirror whether the discharges follow each other quickly or slowly.

In the following experiments it is always assumed that the exhaustion has been carried very far, so that the kathode is surrounded by a dark space :--

If we allow a positive electrode $a$ ( $\beta$ of the figure) to approach a negative plate $k$ ( $\alpha$ of the figure), the positive electrode consisting of a wire which is eut off together with the glass surrounding it, and which dips into the mercury, the following phenomena are observed:-

If $a$ is at a considerable distance from $k$ we have a head of positive light about $a$, and stratifications between $a$ and the glow-rays, of which even the first quite fills the section of the tube and is much curved. If $a$ rises, the layers do not change their position, but one after the other disappear as soon as they touch the positive electrode; this is also the case with the last layer nearest the kathode.

As soon as the end of the wire has passed through this, there is only a small bundle of positive light to be seen upon 
the end of the wire $a$; this also becomes smaller and smaller, without however entirely vanishing; the positive light radiates upwards until the end of the positive electrode has broken through the boundary of the dark negative space about the kathode; then it at once flows downwards like a small luminous waterfall. If the anode rises still higher and approaches the negative plate, the discharge ceases to issue from it and takes place, in highly inconstant fashion, between the upper surface of the mercury-column in the U-tube and $k$. I found thus, as Herr Goldstein has found, that if a positive electrode approaches a negative pole, the end of the positive column remains unchanged. But whilst he asserts that when the positive pole approaches the negative within a distance equal to or less than the interval between the negative pole and the first positive layer of the positive light, at the density employed the positive light disappears altogether, my often repeated experiments showed that there always remains on the positive electrode a little cap of reddish-yellow positive light even when the electrode has much over-passed the end of the positive light; it becomes smaller only gradually. It seems as if this cap, adhering to the positive electrode, were firmly united to it.

Whilst the mode of combination of the positive and negative light can best be followed with electrodes of the form described, certain other phenomena appear much more distinctly if we employ a second plate for the movable positive electrode.

If we allow the anode to approach the kathode, all the phenomena are regular so long as the anode does not penetrate into the dark space about the lrathode, i. e. layer after layer disappears, and when the last has been traversed there remains a small cap of positive light upon the plate, which ascends with it but becomes gradually smaller. If, however, the plate penetrates into the dark space, we always observe a deformation of the negative stratifications, the blue light is driven behind the kathode, whereas it was before only in front, and there forms on the wall a narrow strip of positive light, as also Hittorf * observed upon close approach of anode and kathode. The kathode-rays themselves, which usually proceed from the centre of the kathode, are compressed altogether to the edge of the kathode, from which they issue like a star, whilst the middle of the kathode remains quite dark $\uparrow$; moreover kathode

* Pogg. Ann. vol. cxxxvi. pp. 1, 197 (1869).

$\uparrow$ This experiment shows that the kathode-rays do not always run in the direction of the lines of force. In the case of two parallel plates the lines of force are at right angles to the plates, and in the space between. them the potential falls most rapidly, nevertheless the kathode-rays issue from the edge of the plate. 
and anode attract each other with great force. If they have been made to touch, and then we try to separate them, sparks leap across and the kathode vibrates to and fro.

If the machine works very slowly, then, as soon as the blue negative layer has been traversed, irregular discharges set in, the green light of which is seen at different points of the tube, just as with a tube which has been exhausted so far that the discharges traverse the gas only with difficulty.

A conclusion as to these last phenomena is furnished by experiments in which the poles of the machine are brought within a small distance, some 3 or 5 millim., of each other, and then the lower electrode is allowed to approach the other; as soon as the two electrodes are sufficiently near, a stream of sparks passes between the poles of the machine, whilst the tube remains dark. If, then, the distance of the electrodes is increased, the discharge in the tube suddenly commences, whilst no electricity passes between the poles of the machine. If, now, besides the experimental tube, we include also the comparison-tube in the circuit, then, when sparks are passing between the poles of the machine, we see the walls of the tube shine with bright green phosphorescence at the points where positive light appears in the ordinary passage of the discharge. The reason of this is, that the small distance between the plates of the principal discharge-tube causes this to act as a condenser ; they become gradually charged until suddenly the discharge between the poles of the machine commences, and the whole quantity of (negative) electricity, which is accumulated on the one electrode of the tube, discharges itself suddenly backwards through the small tube, and thereby produces the green light.

It follows from the experiments described that, in highly exhausted spaces, the discharge between a positive and a negative electrode, if their distance apart is less than a certain limit, takes place with greater difficulty the nearer they are to each other. This result was confirmed and completed by the following experiment:-

Two discharge-tubes (figs. 4 and $4 b$ ) were connected at the same time with the pump, which in this case was a Geissler's pump, instead of the Töpler's pump usually employed. The tubes were each 26 millim. wide and 39 millim. long; electrodes of aluminium wire were melted into each, which were covered with glass except about 1 millim. In the tube $a$ the electrodes were 1 millim., in the other tube $b, 20$ millim. apart ; the tubes were included in parallel arc in the circuit of the machine. At high pressures the discharge took place only in $a$; at low pressures of about 5 millim. and less, in both tubes at the same 
time, and at still higher exhaustion only in $a$. When this was the case, if the mercury reservoir of the pump was raised, and the gas present in it thus driven again into the dischargetubes, then the discharge took place again in $b$ and not in $a$. This behaviour at high pressures, and at medium pressures, follows at once from the views ordinarily held : at high pressures the induction exerted by the electrodes upon each other, and on the layer of gas between them, is of importance; at medium pressures the resistance of the positive column of light disappears in comparison with that of the negative light; in fact, at medium pressures the two tubes differ only by having a positive colunin of different length, which exerts no perceptible influence upon the potential which is necessary to the discharge, so that the discharge can take place in both tubes at the same time. At the third pressure mentioned above, the dark space surrounding the kathode has, in the tube in which the electrodes are close together, spread out so far that it completely surrounds the anode. This opposes so great a resistance to the passage of the electricity, that the discharge traverses only the tube in which the electrodes are far apart, in which the dark kathode-space does not yet reach the positive electrode. Upon still further exhaustion the dark kathodespace surrounds the anode also in the tube $b$; and now the discharge must of course take place more easily in the tube in which the electrodes are near each other, since here there is a smaller part of the dark space to be traversed.

From the experiments described above we draw the following two important conclusions :-

(1) The dark kathode-space opposes a very great resistance to the positive discharge.

(2) The union of the positive and negative electricities takes place in the "glow-rays."

Hittorf* had already concluded, from experiments with electrodes near together, that resistances existed in the neighbourhood of the kathode; but since he did not vary the distance of the electrodes from each other, he was not able to determine the position of the resistance, so as to explain the phenomena.

3. Heating of the Gas at different Distances from the Kathode.-A whole series of experiments was undertaken, in order to investigate the decrease of heating-effect in passing from the negative pole towards the positive.

For this purpose a method was employed which has already been used by $G$. Wiedemann and R. Rühlmann $\dagger$ for another purpose. The junction of a thermo-electric element, consisting

* Pogy. Ann. cxxxvi. pp. 1, 197 (1869).

† Pogg. Ann. xlv. p. 35 (1872). 
of two wires of iron and German-silver soldered together, was kept in contact with the outside of the tube by means of a spring, and the deflections read off upon an astatic galvanometer. These give a measure for the heating of the glass tube; and this is nearly proportional to the heating of the portions of gas close at hand. The experiments were always so arranged that the thermo-electric element was moved from the electrode towards the positive light, and back again in the opposite direction; the deflections were only noted after they had become constant.

In the following tables we give the differences of the deflections A observed at the position of the positive light, and at different distances $\mathrm{E}$ from the kathode. The length of the dark kathode-space was taken as measuring the pressure. In the curves the abscissa represent the distances from the kathode, and the ordinates the deflections of the galvanometer.

I. A first series of experiments was made with a thin-walled glass tube 25 millim. wide; the electrodes were circular plates (fig. 5).

Exhaustion high. Dark space 37 millim. Green light faintly visible. Limit of positive light 210 (curve $a$ ).

\begin{tabular}{|r|r|r|r|r|r|}
\hline E. & A. & E. & A. & E. & A. \\
\hline 0 & 27 & 60 & 22 & 210 & 9 \\
13 & 20 & 90 & 23 & 250 & 3 \\
32 & 17 & 122 & 22 & 290 & 0 \\
42 & 17 & 160 & 18 & & \\
\hline
\end{tabular}

Dark space 30 millim. No green light. Limit of positive light at 220 (curve $b$ ).

\begin{tabular}{|c|c|c|c||c|c|}
\hline E. & A. & E. & A. & E. & A. \\
\hline 0 & 19 & 70 & 23 & 190 & 9 \\
11 & 17 & 108 & 20 & 220 & 3 \\
32 & 17 & 160 & 13 & 280 & 0 \\
\hline
\end{tabular}

Dark space 20 millim. No green light. Limit of positive light at 200 (curve c).

\begin{tabular}{|c|c||c|c||c|c|}
\hline E. & $\boldsymbol{A}$. & E. & A. & E. & A. \\
\hline 0 & 23 & 70 & 18 & 140 & 5 \\
20 & 17 & 95 & 13 & 200 & 4 \\
32 & 19 & 105 & 10 & 280 & 0 \\
48 & 17 & & & & \\
\hline
\end{tabular}


Dark space 14 millim. Limit of positive light at 120 (curve $d$ ).

\begin{tabular}{|c|c|c|c|c|c|}
\hline E. & A. & E. & A. & E. & A. \\
\hline 0 & 26 & 40 & 1.2 & 150 & 1 \\
\hline 14 & 22 & 62 & 8 & 260 & 0 \\
\hline 22 & 20 & 100 & 2 & & \\
\hline
\end{tabular}

Dark space 8 millim. Positive light begins at about 75 ( curve $e$ ).

\begin{tabular}{|r|r||c|c||c|c|}
\hline E. & A. & E. & A. & E. & A. \\
\hline 0 & 17 & 52 & 2 & 111 & 1 \\
8 & 11 & 75 & 1 & 125 & 0 \\
26 & 6 & & & & \\
\hline
\end{tabular}

II. Narrower tube 18 millim. wide (fig. 6).

Dark space 14 millim. Green phosphorescent light appeared evon at the edge of the dark space, which, however, cannot be produced by the kathode-rays, since these issue from only a small portion of the kathode, and form a very slightly divergent pencil of rays, which at a distance of 14 millim. certainly does not meet the walls of the tube.

The boundary between positive and negative light cannot be determined. Also there are no stratifications. Fig. $6 f$ gives again the results obtained. The horizontal dimensions are in this diagram twice as great, and the vertical dimensions half as great as in the former ones.

\begin{tabular}{|c|c||c|c||c|c|}
\hline E. & A. & E. & A. & E. & A. \\
\hline 0 & 38 & 44 & 95 & 130 & 11 \\
8 & 47 & 65 & 66 & 170 & 3 \\
14 & 71 & 100 & 25 & 222 & 0 \\
30 & 100 & & & & \\
\hline
\end{tabular}

Dark space about 10 millim. (curve $g$ ).

\begin{tabular}{|r|r||r|r|r|r|}
\hline E. & A. & E. & A. & E. & A. \\
\hline 0 & 51 & 40 & 112 & 130 & 11 \\
10 & 71 & 60 & 86 & 180 & 2 \\
16 & 92 & 100 & 32 & 220 & 0 \\
22 & 102 & & & & \\
\hline
\end{tabular}


Dark space 8 millim. Discharge not stratified (curve $h$ ).

\begin{tabular}{|r|r||c|c||c|c|}
\hline E. & A. & F. & A. & E. & A. \\
\hline 0 & 35 & 25 & 40 & 85 & 0 \\
4 & 32 & 40 & 21 & 120 & 0 \\
12 & 42 & 65 & 5 & & \\
\hline
\end{tabular}

Dark space 4 millim.

\begin{tabular}{|c|c||c|c|c|c|}
\hline E. & A. & E. & A. & E. & A. \\
\hline 10 & 13 & 20 & 7 & 125 & 0 \\
5 & 15 & 70 & 2 & & \\
\hline
\end{tabular}

The curves and numbers belonging to this second tube run exactly similarly to those found for the first tube.

III. Still narrower tube, 11 millim. wide.

Dark space 11 millim.

\begin{tabular}{|r|c||c|c||c|c|}
\hline E. & A. & E. & A. & E. & A. \\
\hline 0 & 37 & 25 & 53 & 110 & 4 \\
6 & 43 & 66 & 20 & 190 & 0 \\
10 & 55 & & & & \\
\hline
\end{tabular}

Dark space 8 millim.

\begin{tabular}{|r|r||c|c|c|c|}
\hline E. & A. & E. & A. & E. & A. \\
\hline 0 & 39 & 23 & 29 & 70 & 9 \\
6 & 44 & 33 & 22 & 110 & 3 \\
13 & 39 & 55 & 7 & & \\
\hline
\end{tabular}

Dark space 6 millim.

\begin{tabular}{|l|l||l|r|r|r|}
\hline E. & A. & E. & A. & E. & A. \\
\hline 0 & 16 & 18 & 19 & 80 & 5 \\
6 & 21 & 40 & 7 & 150 & 0 \\
\hline
\end{tabular}

Dark space 3 millim.

\begin{tabular}{|c|c||c|c||c|c|}
\hline E. & A. & E. & A. & E. & A. \\
\hline 0 & 22 & 15 & 9 & 120 & 0 \\
2 & 19 & 65 & 6 & & \\
\hline
\end{tabular}

Phil. Marg. S. 5. Vol. 18. No. 110. July 1884. 
As with this tube the numbers run exactly as with the others, no curve is given.

All these experiments show that for the low pressures at which the dark kathode space is distinctly formed the following holds good:-

The production of heat in the electric discharge, starting from the positive electrode, rises at first slowly, then rapidly, reaching its maximum within the "glow-rays," from which point it decreases, altaining a relative minimum within the dark space about the kathode, to which, however, corresponds a much higher production of heat than in the positive light. At the kathode itself the production of heat attains a very high value, as shown by separate experiments.

If the pressure is increased, then with decrease of length of the dark kathode-space and shortening of the glow-rays the maximum and minimum of heating-effect move closer together, until at last they can no longer be separated by experimental means.

In order further to prove the peculiar course of the heatingeffects obtained by the methods described and shown by means of the curves, the following arrangement was employed. At the upper end of the usual Hoat carrying the electrode (Pl. II. fig. 1) a thermometer was attached, having its scale below, as shown in fig. 7. The mercury reservoir $a$ is cylindrical, 5 millim. broad and 17 millim. long. It is protected against the kathode-rays by a horizontal screen $b$ of mica carried by a pair of platinum wires c. But as these measurements are concerned, not with the temperatures of the gas, but with the quantities of heat produced in the unit time, the rise in temperature in equal times must be observed when the thermometer is placed at different points of the tube. We must, of course, also apply corrections for radiation and conduction : this was done by observations before and after breaking the current in the usual way. The measurements were carried out with a tube of about 140 millim. width and 500 millim. long. The dark space had a length of about 35 millim.

The following table gives the numbers found in three series (I., II., III.) of experiments. The different series are not comparable with each other as to absolute value.

Edenotes the distance of the electrode; $W$ the observed rise in temperature in arbitrary units (generally $\frac{1}{100} 0^{\circ}$ per minute for a current producing 100 millim. deflection). 


\begin{tabular}{|r|r||r|r||r|r|}
\hline \multicolumn{2}{|c|}{ I. } & \multicolumn{2}{c|}{ Ir. } \\
\hline E. & W. & E. & W. & III. \\
\hline 170 & W. & W. \\
\hline 135 & 68 & 160 & 100 & 170 & 60 \\
85 & 72 & 130 & 112 & 40 & 125 \\
55 & 93 & 82 & 110 & 20 & 40 \\
32 & 78 & 60 & 100 & 10 & 48 \\
20 & 73 & 30 & 62 & & \\
11 & 45 & 14 & 20 & & \\
5 & 45 & & & & \\
\hline
\end{tabular}

When the mica shield touched the electrode, a very great rise was observed.

The course of production of heat, as given by measurements with the thermometer, is thus exactly the same as that furnished by measurements with the thermo-electric element. From a minimum in the positive discharge it rises to a maximum, then sinks to a minimum, finally attaining a second maximum at the kathode itself.

The causes of these peculiarities in heating-effect cannot be determined without further experiment, but in the meantime we may note the following points.

The following equation holds good for any body traversed by the current

$$
\frac{\partial^{2} V}{\partial x^{2}}+\frac{\partial^{2} V}{\partial y^{2}}+\frac{\partial^{2} V}{\partial z^{2}}=-4 \pi \rho
$$

where $\nabla$ denotes the potential at any point and $\rho$ the density at the same point. If we have a tube (whose axis is the axis of $x$ ) which the current fills uniformly, then

$$
\frac{\partial^{2} V}{\partial y^{2}}=\frac{\partial^{2} V}{\partial z^{2}}=0 ; \text { consequently } \frac{\partial^{2} V}{\partial x^{2}}=-4 \pi \rho .
$$

In ordinary conductors $\rho=0:$ hence $\frac{\partial V}{\partial x}$ is constant, or, for unit length, $V_{0}-V_{1}=$ constant, being proportional to the resistance $w$. Hence if $a$ denote an absolute constant,

$$
\mathrm{V}_{0}-\mathrm{V}_{1}=a w
$$

Hence, with equal resistances, there must be equal productions of heat in equal lengths. If we find that the heat-production at different points of a tube is not constant, this is explained, if $\rho=0$, by the fact that the resistance at different points of the tube is very different.

We have seen that close to the electrode we have a very E 2 
large production of heat, and that this decreases, reaching a minimum within the dark kathode-space, then it rises again to a maximum within the glow-rays, and sinks again towards the positive light.

It is therefore, according to our previous experiments, not possible to deduce the change in heating-effect from the change in resistance. We have not the maximum heating exactly at those places where the greatest resistance is offered to the radiation of the positive light. There are now two things possible, either (1) $\rho$ is not zero-that is, free electricity moves within the tube; or (2) a part of the fall in potential is used up at those points where the heating-effect is a minimum in imparting to the electricity a certain kinetic energy, which disappears again at the points of maximum heating-effect.

I shall endeavour to determine which of these two views is the correct one by special measurements of potential within the tube.

4. Deflection of the Positive Column of Light.-Two circumstances are of importance in connexion with the deflection of the sensitive positive light, which takes place upon touching the tube traversed by the discharge with the finger ; it sometimes consists in an irregular attraction, sometimes in a symmetrical deflection.

The one circumstance is the change in the distribution of free electricity upon the wall of the glass tube, by which the potential is lowered at the point touched, and the discharge is deflected towards the point touched; this is especially the case at high pressures. The second circumstance is the formation of a dark space at the point where positive electricity flows into a conductor, or becomes bound. The experiments with the floating electrode show, indeed, that the passage of the positive electricity through the dark space meets with great resistance, and then the discharge bends round backwards. But so soon as any point of the wall is put into connexion with the ground, a kathode is produced at that point, kathode-rays issue from it, and the peculiar reddish light is seen; consequently a dark space must form around this point, which prevents a portion of the tube from being traversed by the positive light, round which the discharge therefore bends.

Exactly similar reasons explain the luminosity of the tube when, the discharge being such that the tube remains dark, it is encircled by the fingers, or by a ring of tin-foil. Then the whole woll at the points touched becomes a kathode, and opposes to the discharge a great resistance, which is, of course, attended by a loss of energy, and has for consequence 
a brighter luminosity of the gas. To the same cause I should be disposed to refer the great heating, otherwise so remarkable, in a calorimeter surrounding the discharge-tube, when a powerful discharge is sent through, and a good conducting fluid in connexion with the earth is employed in the calorimeter. The quantities of electricity, which become bound, become greater, especially where air-sparks are included, and consequently the formation of the dark space is much facilitated, so that the resistance offered to the passage of the discharge must increase in a high degree.

5. Influence of a powerful Magnet upon the Discharge.-The results which we have obtained also explain the phenomena which take place when the discharge is subjected to the action of a powerful magnet. The best form of magnet to employ is a cylindrical magnet, magnetized by a spiral of wire. It is known that under the influence of such a magnet the positive light is thrown into curves, which correspond nearly to those assumed by a solid flexible conductor fixed at both ends, whilst the glow-rays behave like a conductor fixed at one end. In a wide, strongly exhausted tube the stratified discharge was obtained, and then one pole of the electromagnet described was moved along gradually from the positive pole-plate $a$. 'The well-known forms appeared in the positive light; the discharge appeared continuous in the revolving mirror. If we bring the magnet nearer to the negative pole, the glow-rays become compressed at the side, as may be seen from the green light which appears along the wall, and the positive light with its stratifications is seen to move, somewhat deformed it is true, in the space vacated by the glow-rays. The form of the discharge is represented in fig. 3. The bend at $b$ corresponds to the magnet beneath.

If the magnet is brought still nearer to the kathode, and so far that the tongue a reaches to the limit of the dark kathodespace, it suddenly falls down and unites with glow-rays at the point where they strike the wall, and the discharges become discontinuous; so that in this case a much higher potential is necessary for the discharge than in the first case, or a considerable resistance is produced.

These experiments appear to me to show that the formation and position of the positive light does not depend so much on the position of the positive and negative polas, but rather upon the formation of the glow-rays issuing from the negative pole, and that generally, in accordance with their formation and position, the discharge shows a greater or less degree of discontinuity.

The above observation of the transformation of the con- 
tinuous form of discharge into the discontinuous agrees entirely with the results previously abtained by approaching the positive to the negative pole. If we denote as the positive direction of the glow-rays that which issues from the bright kathode-layer, many discharges occurred so long as a union of the positive electricity could take place in the negative direction with the glow-rays; but as soon as that was no longer the case, much fewer discharges occurred. Exactly the same is the case here, so long as the glow-rays have not all been deflected from the axis of the tube to the side; the positive light can still unite with them in the negative direction. But as soon as a complete deflection has taken place, we have exactly the same case as if the positive electrode were behind the bright kathode-layer, and the discharge becomes continuous.

[To be eontínued.]

IV. On Molecular Latent Heat. By Frederick Trodton, Trinity College, Dublin*.

( $)^{\mathrm{N}}$ comparing the quantities of heat necessary to evaporate at constant pressure quantities of different liquids taken in the ratio of their molecular weights, it is found that the amount of heat required by any body is approximately proportional to its absolute temperature at the point of ebullition. For example, the latent heat of bromine is 45.9 , it boils at $63^{\circ}$, and the density of the body is $791 \cdot 75$. The latent heat of butyric acid is $114 \cdot 7$, it boils at $162^{\circ}$, and its density is 44 . The quantities of heat required to evaporate quantities of the bodies in the ratio of their molecular weights is obtained by multiplying the latent heat by the density; and the quantities thus obtained bave an approximately constant ratio to the absolute temperatures of the boiling-points, thus :-

$$
\frac{45 \cdot 9 \times 79 \cdot 75}{273+63^{\circ}}=10 \cdot 89, \quad \frac{114 \cdot 6 \times 44}{273+162^{\circ}}=11.59 \text {. }
$$

This ratio is nearly constant for most bodies, but is still more nearly so for those bodies which are chemically related to each other.

The relation, then, may be put into this simple form. The molecules of chemically related bodies, in changing from the gaseous to the liquid state at the same pressure, disengage quantities of heat, which may be called the molecularlatent heat, directly proportional to the absolute temperature of the point of ebullition. The similarity is very striking

* Communicated by the Author. 